\title{
Article \\ Characterization of Almost Yamabe Solitons and Gradient Almost Yamabe Solitons with Conformal Vector Fields
}

\author{
Ali H. Alkhaldi 1,+ ${ }^{\text {, Pişcoran Laurian-Ioan }}{ }^{2, *,+}$, Abimbola Abolarinwa ${ }^{3,+}$ (D) and Akram Ali ${ }^{1,+}$ \\ 1 Department of Mathematics, College of Science, King Khalid University, Abha 61413, Saudi Arabia; \\ ahalkhaldi@kku.edu.sa (A.H.A.); akali@kku.edu.sa (A.A.) \\ 2 North University Center of Baia Mare, Department of Mathematics and Computer Science, Technical \\ University of Cluj Napoca, Victoriei 76, 430122 Baia Mare, Romania \\ 3 Department of Mathematics, University of Lagos, Akoka, Lagos 101017, Nigeria; a.abolarinwa1@gmail.com \\ or aabolarinwa@unilag.edu.ng \\ * Correspondence: Laurian.PISCORAN@mi.utcluj.ro \\ + These authors contributed equally to this work.
}

Citation: Alkhaldi, A.H.;

Laurian-Ioan, P.; Abolarinwa, A.; Ali, A. Characterization of Almost Yamabe Solitons and Gradient Almost Yamabe Solitons with Conformal Vector Fields. Symmetry 2021, 13, 2362. https://doi.org/ $10.3390 /$ sym 13122362

Academic Editor: Abraham A. Ungar

Received: 15 October 2021

Accepted: 20 November 2021

Published: 8 December 2021

Publisher's Note: MDPI stays neutral with regard to jurisdictional claims in published maps and institutional affiliations.

Copyright: (c) 2021 by the authors. Licensee MDPI, Basel, Switzerland. This article is an open access article distributed under the terms and conditions of the Creative Commons Attribution (CC BY) license (https:// creativecommons.org/licenses/by/ $4.0 /)$.
Abstract: In this paper, some sufficient conditions of almost Yamabe solitons are established, such that the solitons are Yamabe metrics, by which we mean metrics of constant scalar curvature. This is achieved by imposing fewer topological constraints. The properties of the conformal vector fields are exploited for the purpose of establishing various necessary criteria on the soliton vector fields of gradient almost Yamabe solitons so as to obtain Yamabe metrics.

Keywords: conformal vector fields; gradient almost Yamabe solitons; scalar curvatures

\section{Introduction}

The notion of Yamabe flow was established by R. Hamilton [1] on a Riemannian manifold $\mathcal{M}^{n}$ of dimension $n$, in which the given family of time-dependent metrics $g(t)$ is warped by development according to the governing equation

$$
\frac{\partial}{\partial t} g(t)=-\mathcal{R}(t) g(t)
$$

where $\mathcal{R}(t)$ denotes the scalar curvature of $g(t)$. It is well known that this flow exists globally and converges to a fixed point of constant scalar curvature in the conformal class [1-3]. Yamabe solitons naturally carry detailed descriptions of singularities which arise in Yamabe flow, meaning that they represent auto-identical solutions of (1) in the conformal class (self-similar conformal solutions). We also know that Yamabe flow and Ricci flow coincide when the dimension of the underlying manifold is 2; thus, in this case, a Yamabe soliton is a Ricci soliton. This is not the case in general, since Yamabe flow preserves the conformal class of $g(t)$, while the Ricci flow does not for $n>2$. Let $\zeta$ be a complete smooth vector field and $\lambda$ be a scalar. A quadruple $(\mathcal{M}, g, \zeta, \lambda)$ of a connected $n$-dimensional Riemannian manifold together with $\zeta$ and $\lambda$ is called a Yamabe soliton if it satisfies the following equation:

$$
\frac{1}{2} \mathcal{L}_{\zeta} g=(\mathcal{R}-\lambda) g
$$

where $\mathcal{L}_{\zeta}$ represents the Lie derivative in the direction of $\zeta$. The vector field $\zeta$ in the definition above is referred to as a soliton vector field and $\lambda$ as a soliton constant for $(\mathcal{M}, g)$. If the soliton vector field $\zeta$ is the gradient of a smooth function, then a Yamabe soliton is considered a gradient Yamabe soliton. Let us remark that in [4,5], solitons are studied. 
Now we consider a generalized situation when $\lambda: \mathcal{M} \longrightarrow \mathbb{R}$ is a smooth variable function. In this case the quadruple $(\mathcal{M}, g, \zeta, \lambda)$ is known as an almost Yamabe soliton if it recognizes a smooth function $\lambda$ into (2); that is,

$$
\frac{1}{2} \mathcal{L}_{\zeta} g=(\mathcal{R}-\lambda) g .
$$

In the sequel, a Yamabe soliton (resp. almost Yamabe soliton) will be classified in accordance to whether the soliton constant (resp. soliton function) $\lambda$ is negative, null or positive. That is, it is considered to be expanding if $\lambda<0$, steady if $\lambda=0$ and shrinking if $\lambda>0$; otherwise, they are referred to as indefinite for the case where $\lambda$ is a variable function. Almost Yamabe solitons as introduced in the famous paper [6] represent a natural extension of the Yamabe solitons [1] and Yamabe metrics [7]. Let $f$ be a real valued function on $M$, and suppose that $\zeta=\nabla f$ for a gradient $f$. Then $(\mathcal{M}, g, f, \lambda)$ is called a gradient almost Yamabe soliton, and (3) then reads as follows:

$$
\operatorname{Hess}(f)=(\mathcal{R}-\lambda) g,
$$

where $\operatorname{Hess}(f)$ is the Hessian of function $f$. It should be noted that the soliton will be called trivial if $f$ is a constant function on $M$, or the vector field $\zeta$ or $\nabla f$ is Killing.

Almost Yamabe solitons also serve as particular solutions to (1) in [1] and they are fixed points of the Yamabe flow modulo diffeomorphisms and scalings of Riemannian metrics. In this sense, understanding the behavior of the Yamabe flow requires an awareness of the analytical and geometrical characteristics of almost Yamabe solitons. Combining the above facts, it is clear that $g(t)$ will be a Yamabe metric on $M$ if $\mathcal{R}$ is a constant scalar curvature. Finding conditions on Yamabe solitons' vector fields so that their metrics possess constant scalar curvature is one of the most fascinating topics in Yamabe solitons' geometry. For such a study, there are two options: imposing stronger topological limits with smaller geometric and analytic conditions, or imposing reduced topological restrictions with more analytic and geometric restrictions. The present research looks into Yamabe solitons from the second perspective, focusing solely on the linked topological restriction. Then, on the vector fields of the Yamabe solitons, we prove numerous new sufficient conditions for their metrics to be Yamabe.

\section{Preliminaries}

Let $(\mathcal{M}, g)$ be a Riemannian manifold. It should be recalled that a smooth vector field $\zeta$ on $(\mathcal{M}, g)$ is said to be a conformal if the local flow of $\zeta$ is composed of local conformal transformations of $(\mathcal{M}, g)$, which commensurate with the notion that the vector field $\zeta$ is adequate; that is,

$$
\frac{1}{2} \mathcal{L}_{\zeta} g=\beta g,
$$

where $\beta$ lies in $C^{\infty}(\mathcal{M})$ and is called the potential function of $U$. Many theoretical aspects of conformal and homothetic ( $\beta=$ const) transformations can be found, for example in [8,9]. Utilizing Koszul's formula $[10,11]$, we obtain the following for a vector field $\zeta$ on $\mathcal{M}$ :

$$
2 g\left(\nabla_{U} \zeta, V\right)=\left(\mathcal{L}_{\zeta} g\right)(U, V)+d \eta(U, V),
$$

for all $U, V \in \Gamma(T \mathcal{M})$, where $\eta$ denotes the 1-dual form to $\zeta$, which has the relation $\eta(U)=g(U, \zeta)$. Classifying skew symmetric tensor fields $\varphi$ of type one-one on $\mathcal{M}$,

$$
d \eta(U, V)=2 g(\varphi U, V),
$$


where $d$ is a differential operator and $\eta$ is a 1-dual form. Now, using (2), (6) and (7), we get the following equation:

$$
\nabla_{U} \zeta=(\mathcal{R}-\lambda) U+\varphi U
$$

With the help of Equation (8), a direct calculation results in the next equation with respect to the Riemannian curvature tensor of the almost Yamabe soliton

$$
\mathcal{R}(U, V) \zeta=U(\mathcal{R}-\lambda) V-V(\mathcal{R}-\lambda) U+(\nabla \varphi)(U, V)-(\nabla \varphi)(V, U),
$$

such that the covariant derivative of $\varphi$ is defined as

$$
(\nabla \varphi)(U, V)=\nabla_{U} \varphi V-\varphi \nabla_{U} V,
$$

with a closed 2-form $\Omega$ such that $\Omega(U, V)=g(\varphi U, V)$. Taking advantage of (9), one direct calculation leads to the following:

$$
(\nabla \varphi)(U, V)=\mathcal{R}(U, \zeta) V+V(\mathcal{R}-\lambda) U-g(U, V) \nabla(\mathcal{R}-\lambda),
$$

where $\nabla \mathcal{R}$ stands for the gradient of the scalar curvature $\mathcal{R}$. The Ricci operator $\mathcal{Q}$ of the Yamabe soliton is defined as

$$
g(\mathcal{Q} U, V)=\operatorname{Ric}(U, V),
$$

where Ric denotes the Ricci tensor of an almost Yamabe soliton $(\mathcal{M}, \lambda, \zeta, g)$. As $\mathcal{Q}$ is symmetric, then we have

$$
\sum(\nabla \mathcal{Q})=\frac{1}{2} \nabla \mathcal{R}
$$

where $\left\{e_{1}, e_{2}, \cdot, e_{n}\right\}$ denotes a local orthonormal frame with respect to an almost Yamabe soliton $(\mathcal{M}, \lambda, \zeta, g)$. Applying the skew symmetric properties of the operator $\varphi$ and utilizing (9), we derive

$$
\operatorname{Ric}(V, \zeta)=-(n-1) V(\mathcal{R}-\lambda)-g\left(V, \sum(\nabla \varphi)\left(e_{i}, e_{i}\right)\right),
$$

which is equivalent to the following

$$
\mathcal{Q}(\zeta)=-(n-1) \nabla(\mathcal{R}-\lambda)-\sum(\nabla \varphi)\left(e_{i}, e_{i}\right) .
$$

An operator $\Delta: \Gamma(T \mathcal{M}) \longrightarrow \Gamma(T \mathcal{M})$ is called a Laplace operator and defined as

$$
\Delta U=\sum\left(\nabla_{e_{i}} \nabla_{e_{i}} U, \nabla_{\nabla_{e_{i}}} e_{i} U\right) .
$$

Therefore, using (8), we have

$$
\Delta \zeta=\nabla(\mathcal{R}-\lambda)+\sum(\nabla \varphi)\left(e_{i}, e_{i}\right) .
$$

\section{Main Results}

Theorem 1. If the scalar curvature $\mathcal{R}$ and the soliton field $\zeta$ satisfy the following conditions

$$
\mathcal{Q}(\zeta)+\Delta(\zeta)=v \zeta, \quad \text { and }, \quad \frac{n-2}{2 v}\left(\frac{g(\nabla \lambda, \nabla \mathcal{R})}{\mathcal{R}-\lambda}\right)+\mathcal{R} \leq\left(\frac{n(n-1)}{n-2}\right) v,
$$

respectively, for an $n$-dimensional connected almost Yamabe soliton $(\mathcal{M}, \lambda, \zeta, g)$ with $n>2$, then the scalar curvature $\mathcal{R}$ of an almost Yamabe soliton $(\mathcal{M}, \lambda, \zeta, g)$ is constant. 
Proof. Utilizing Equations (14) and (15), we provide

$$
\mathcal{Q}(\zeta)+\Delta(\zeta)=-(n-2) \nabla(\mathcal{R}-\lambda) .
$$

This implies the following by using $\mathcal{Q}(\zeta)+\Delta(\zeta)=v \zeta$

$$
\nabla(\mathcal{R}-\lambda)=-\frac{v \zeta}{n-2} .
$$

The Hessian $A_{\mathcal{R}}$ operator of the scalar curvature $\mathcal{R}$ is computed by using (8) and (17) as

$$
A_{\mathcal{R}} U=-\frac{v(\mathcal{R}-\lambda)}{n-2} U-\frac{v}{n-2} \varphi U+\nabla_{U} \nabla \lambda .
$$

The last expression leads to the following:

$$
A_{\mathcal{R}} U+\frac{v(\mathcal{R}-\lambda)}{n-2} U-\nabla_{U} \nabla \lambda=\frac{v}{n-2} \varphi U .
$$

Observe that the left-hand side of the last equation is symmetrical, while the right-hand side is skew symmetrical; as a result, we obtain

$$
A_{\mathcal{R}} U=-\frac{v(\mathcal{R}-\lambda)}{n-2} U+\nabla_{U} \nabla \lambda \text { and } \varphi U=0 .
$$

Taking advantage of the final conclusion that $\varphi=0$ in (14), one can derive

$$
\mathcal{Q}(\zeta)=-(n-1) \nabla(\mathcal{R}-\lambda) .
$$

The divergence of $\mathcal{Q}(\zeta)$ is defined as

$$
\begin{aligned}
\operatorname{div}(\mathcal{Q}(\zeta)) & =\sum_{i=1}^{n} g\left(\nabla_{e_{i}} \mathcal{Q}(\zeta), e_{i}\right) \\
& =\sum_{i=1}^{n} e_{i} g\left(\zeta, \mathcal{Q}\left(e_{i}\right)\right) \\
& =\sum_{i=1}^{n}\left(g \nabla_{e_{i}} \zeta, \mathcal{Q}\left(e_{i}\right)\right)+\sum_{i=1}^{n} g\left(\zeta,(\nabla \mathcal{Q})\left(e_{i}, e_{i}\right)\right) \\
& =\sum_{i=1}^{n}\left((\mathcal{R}-\lambda) e_{i}+\varphi e_{i}, \mathcal{Q}\left(e_{i}\right)\right)+\frac{1}{2} \zeta(\mathcal{R}) .
\end{aligned}
$$

Applying the skew-symmetry property of the tensor $\varphi$ and a local orthonormal frame that diagonalizes the symmetric operator $\mathcal{Q}$, we arrive at $\sum_{i=1}^{n} g\left(\varphi e_{i}, \mathcal{Q}\left(e_{i}\right)\right)=0$, which, when combined with the preceding equation, provides

$$
\operatorname{div}(\mathcal{Q}(\zeta))=(\mathcal{R}-\lambda) \mathcal{R}+\frac{1}{2} \zeta(\mathcal{R})
$$

Taking the divergence of both sides in the Equation (19) and using (20), we provide

$$
(\mathcal{R}-\lambda) \mathcal{R}+\frac{1}{2} g(\nabla \mathcal{R}, \zeta)=-(n-1) \Delta(\mathcal{R}-\lambda)
$$

Inserting (17) in (21), we get

$$
(\mathcal{R}-\lambda) \mathcal{R}-\frac{n-2}{2 v}\|\nabla \mathcal{R}\|^{2}+\frac{n-2}{2 v} g(\nabla \lambda, \nabla \mathcal{R})=-(n-1) \Delta(\mathcal{R}-\lambda) .
$$


Taking the trace of (18) for computing the term $\Delta(\mathcal{R}-\lambda)$, and then using it in the above equation, we get

$$
(\mathcal{R}-\lambda) \mathcal{R}-\frac{n-2}{2 v}\|\nabla \mathcal{R}\|^{2}+\frac{n-2}{2 v} g(\nabla \lambda, \nabla \mathcal{R})=\frac{n(n-1) v}{n-2}(\mathcal{R}-\lambda) .
$$

The above equation leads to the following:

$$
(\mathcal{R}-\lambda)\left(\mathcal{R}-\frac{n(n-1) v}{n-2}\right)+\frac{n-2}{2 v} g(\nabla \lambda, \nabla \mathcal{R})=\frac{n-2}{2 v}\|\nabla \mathcal{R}\|^{2} .
$$

By hypothesis of theorem, our assumption holds; we get $\|\nabla \mathcal{R}\|^{2} \leq 0$ as $n>2$ and we finally obtain $\nabla \mathcal{R}=0$. This implies that $\mathcal{R}$ is constant. This competes the proof of theorem.

Theorem 2. Assume that the soliton field $\zeta$ annihilates the Hessian operator $A_{\mathcal{R}-\lambda}$, which satisfies $\Delta \zeta=-v \zeta$ for positive constant $v$ of an $(n>2)$-dimensional connected gradient almost Yamabe soliton $(\mathcal{M}, \lambda, \zeta, g, \nabla f)$. If the Ricci curvature in the direction of the soliton field is a constant, then the Laplacian of the potential function is constant.

Proof. As we assumed that the Ricci curvature is constant with respect to $\zeta$, then $\operatorname{Ric}(\zeta, \zeta)=$ $\epsilon|\zeta|^{2}$, where $\epsilon$ is constant. Utilizing (14) and (15) with $\Delta \zeta=-v \zeta$, we get

$$
\mathcal{Q}(\zeta)=v \zeta-(n-2) \nabla(\mathcal{R}-\lambda)
$$

Taking an inner product with $\zeta$ in the proceeding equation, we derive

$$
(\nu-\epsilon)\|\zeta\|^{2}=(n-2) g(\zeta, \nabla(\mathcal{R}-\lambda)) .
$$

Taking the co-variant derivative along $U$ in above equation, we obtain

$$
2(\nu-\epsilon) g\left(\nabla_{U} \zeta, \zeta\right)=(n-2) g\left(\nabla_{U} \zeta, \nabla(\mathcal{R}-\lambda)\right)+g\left(\zeta, \nabla_{U} \nabla(\mathcal{R}-\lambda) .\right.
$$

As $\zeta$ is annihilated to $A_{\mathcal{R}-\lambda}$, then we get

$$
2(\nu-\epsilon) g\left(\nabla_{U} \zeta, \zeta\right)=(n-2) g\left(\nabla_{U} \zeta, \nabla(\mathcal{R}-\lambda)\right)
$$

Inserting (8) in the above equation and simplifying that, we obtain

$$
\begin{aligned}
2(\nu-\epsilon)(\mathcal{R}-\lambda) \zeta & -(n-2) \nabla(\mathcal{R}-\lambda) \\
= & \varphi\{2(\nu-\epsilon)(\mathcal{R}-\lambda) \zeta-(n-2) \nabla(\mathcal{R}-\lambda)\} .
\end{aligned}
$$

Applying the inner product property with the term $2(\nu-\epsilon)(\mathcal{R}-\lambda) \zeta-(n-2) \nabla(\mathcal{R}-\lambda)$ in (23) and implementing the skew symmetric property of $\varphi$, we derive

$$
\nabla(\mathcal{R}-\lambda)=\left\{\frac{2(\nu-\epsilon)(\mathcal{R}-\lambda)}{n-2}\right\} \zeta
$$

This implies the following:

$$
\nabla(\mathcal{R}-\lambda)=h \zeta
$$

where $h=\left\{\frac{2(v-\epsilon)(\mathcal{R}-\lambda)}{n-2}\right\}$. Applying the co-variant derivative in (24) with respect to $U$, we obtain

$$
A_{\mathcal{R}-\lambda} U=\left\{\frac{2(\nu-\epsilon)}{n-2}\right\} U((\mathcal{R}-\lambda)) \zeta+h(\mathcal{R}-\lambda) U+h \varphi U
$$


The symmetric property of $A_{\mathcal{R}-\lambda}$ gives

$$
\begin{aligned}
2 h \varphi U & =\left\{\frac{2(\nu-\epsilon)}{n-2}\right\}(g(U, \zeta) \nabla(\mathcal{R}-\lambda)-g(U, \nabla(\mathcal{R}-\lambda)) \zeta) \\
& =\left(\frac{(v-\epsilon)}{n-2}\right)^{2}(\mathcal{R}-\lambda)(g(U, \zeta) \zeta-g(U, \zeta) \zeta) \\
& =0 .
\end{aligned}
$$

This means that either $\varphi=0$ or $h=0$. Meanwhile, if $h=0$, we get from (24) that

$$
(\mathcal{R}-\lambda)=A
$$

where $A$ is constant. Now tracing the fundamental Equation (4), we have

$$
\Delta f=(\mathcal{R}-\lambda) n
$$

Joining (26) and (27), we conclude that the Laplacian $\Delta$ of the potential function $f$ is constant. Now for the second case, if we assume that $\varphi=0$, we get from (14)

$$
\mathcal{Q}(\zeta)=-(n-1) \nabla(\mathcal{R}-\lambda)
$$

Taking the inner product with $\zeta$ and using (12), we arrive at

$$
\epsilon\|\zeta\|^{2}=-(n-1)(\zeta(\mathcal{R}-\lambda)) .
$$

Combining Equations (24) and (28), we derive

$$
\left(\epsilon+\frac{2(n-1)(v-\epsilon)(\mathcal{R}-\lambda)}{n-2}\right)\|\zeta\|^{2}=0 .
$$

From the above equation, if we choose $v=\epsilon$, then we conclude from (24), (26) and (27) that the Laplacian of $f$ (the potential function) is again constant. If we choose $\zeta=0$, then Equations (24), (26) and (27) give the same conclusion. Now, we consider $v \neq \epsilon$ and $\zeta \neq 0$; then we have

$$
\mathcal{R}-\lambda=-\frac{\epsilon(n-2)}{2(n-1)(v-\epsilon)} .
$$

It should be noticed that the right-hand side in (30) is constant; then, $\mathcal{R}-\lambda$ is also constant. Hence, we finally reach the conclusion that the Laplacian $\Delta$ of function $f$ is constant with our assumption. This competes the proof of theorem.

Theorem 3. Let $(\mathcal{M}, \lambda, \zeta, g, \nabla f)$ be a connected $n$-dimensional gradient almost Yamabe soliton with $n>2$. If the soliton field $\zeta$ satisfies $\Delta \zeta=-v \zeta$ and $\mathcal{Q}(\zeta)=\epsilon \zeta$ for constants $\nu \geq 0$ and $\epsilon$, then the gradient almost Yamabe soliton is not blue in the domain of a round sphere; if the scalar curvature is constant, then the gradient almost Yamabe soliton is a gradient Yamabe soliton.

Proof. Implementing the Equations (14) and (15), we have

$$
\mathcal{Q}(\zeta)+\Delta \zeta=-(n-2) \nabla(\mathcal{R}-\lambda)
$$

which implies that

$$
\nabla(\mathcal{R}-\lambda)=\frac{v-\epsilon}{n-2} \zeta
$$


Using Equation (8) and the co-variant derivative in the previous equation with respect to $U$, we get

$$
A_{\mathcal{R}-\lambda} U-\frac{(\nu-\epsilon)(\mathcal{R}-\lambda) U}{n-2}-\frac{(\nu-\epsilon) U}{n-2}=0 .
$$

Comparing the symmetric and skew symmetric parts in both sides, then we derive

$$
\begin{aligned}
& \epsilon \zeta=-(n-1) \nabla(\mathcal{R}-\lambda) \\
& v \zeta=-\nabla(\mathcal{R}-\lambda)
\end{aligned}
$$

which is equivalent to the following:

$$
\epsilon=-(n-1) v
$$

Substituting $\epsilon$ in (31) and in $\mathcal{Q}(\zeta)=\epsilon \zeta$, respectively,

$$
\begin{aligned}
\nabla(\mathcal{R}-\lambda) & =-v \zeta \\
\mathcal{Q}(\zeta) & =(n-1) v \zeta
\end{aligned}
$$

If we consider $v=0$, then we get $\nabla(\mathcal{R}-\lambda)=0$, and using (27), we finish with the Laplacian of the potential function $f$ being a constant. Hence, $(\mathcal{M}, \lambda, \zeta, g, \nabla f)$ is not a round sphere. Therefore, we will now use $v>0$. Taking the divergence in the second part of Equation (33), and utilizing (8) and (13), we obtain

$$
\mathcal{R}(\mathcal{R}-\lambda)+\frac{1}{2} \zeta(\mathcal{R})=n(n-1) v(\mathcal{R}-\lambda) .
$$

Multiplying above by $-v$, we derive

$$
-v \mathcal{R}(\mathcal{R}-\lambda)-\frac{v}{2} g(\nabla \mathcal{R}, \zeta)=-n(n-1) v^{2}(\mathcal{R}-\lambda)
$$

Inserting the first part of Equation (33) in (34), one derives

$$
v(\mathcal{R}-\lambda)(n(n-1) v-\mathcal{R})+\frac{1}{2}\|\nabla \mathcal{R}\|^{2}+\frac{1}{2} g(\nabla \mathcal{R}, \nabla \lambda)=0 .
$$

If we assume that the scalar curvature is constant, then $\nabla \mathcal{R}=0$. Therefore, the proceeding equation is equal to the following:

$$
v(\mathcal{R}-\lambda)(n(n-1) v-\mathcal{R})=0
$$

There are two possibilities: $n(n-1) v=\mathcal{R}$ or $(\mathcal{R}-\lambda)=0$. If $\mathcal{R}=n(n-1) v$, then it is obvious. If $\mathcal{R}=\lambda$, then it leads to a contradiction, because $\lambda$ is a smooth function; therefore, the gradient almost Yamabe soliton is reduced to a gradient Yamabe soliton.

\section{Conclusions}

The preceding studies show that by selecting a suitable soliton vector field $[10,12-16]$, we can achieve important geometric findings. In this regard, a vector field created by the gradient of the height function from the immersion has already proven to be a rich source for producing examples of soliton fields. Gradient almost Yamabe solitons, in which the height function is chosen as the potential, may be intriguing for further exploration based on recent work. Furthermore, it appears that considering immersions into a sufficiently large family of manifolds, which include spaces with constant sectional curvature, is a handy way to expand the previous studies to a broader class of ambient spaces. Warped product metrics explain a natural metric that includes spaces with constant sectional curvature throughout its range [17-26]. 
Author Contributions: Conceptualization, A.H.A., P.L.-I., A.A. (Abimbola Abolarinwa) and A.A. (Akram Ali); methodology, A.H.A., P.L.-I., A.A. (Abimbola Abolarinwa) and A.H.A.; writingoriginal draft preparation, A.H.A., P.L.-I., A.A. (Abimbola Abolarinwa) and A.H.A.; writing-review and editing, A.H.A., P.L.-I., A.A. (Akram Ali) and A.H.A. All authors have read and agreed to the published version of the manuscript.

Funding: The authors extend their appreciation to the Deanship of Scientific Research at King Khalid University for funding this work through the research groups program under grant number R.G.P.2/74/42.

Institutional Review Board Statement: Not applicable.

Informed Consent Statement: Not applicable.

Data Availability Statement: There is no data use for this manuscript.

Conflicts of Interest: The authors declare no competing interest.

\section{References}

1. Hamilton, R.S. The Ricci flow on surfaces. In Mathematics and general relativity, Proceedings of the AMS-IMS-SIAM Joint Summer Research Conference in the Mathematical Sciences on Mathematics in General Relativity, Santa Cruz, CA, USA, 22-28 June 1986; American Mathematical Society: Providence, RI, USA, 1988; pp. 237-262.

2. Brendle, S. Convergence of the Yamabe flow for arbitrary initial energy. J. Differ. Geom. 2005, 69, 217-278. [CrossRef]

3. Brendle, S. Convergence of the Yamabe flow in dimension 6 and higher. Invent. Math. 2007, 170, 541-576. [CrossRef]

4. Stepanov, S.; Tsyganok, I. The theory of infinitesimal harmonic transformations and its applications to the global geometry of Riemann solitons. Balkan J. Geom. Appl. 2019, 24, 113-121.

5. Stepanov, S.; Shandra, I. New characteristics of infinitesimal isometry and Ricci solitons. Math. Notes 2012, 92, 422-425. [CrossRef]

6. Barbosa, E.; Ribeiro, E. On conformal solutions of the Yamabe flow. Arch. Der Math. 2013, 101, 79-89. [CrossRef]

7. Yamabe, H. On a deformation of Riemannian structures on compact manifolds. Osaka Math. J. 1960, 12, 21-37.

8. Egorov, I.P. Automorphisms in generalized spaces. J. Sov. Math. 1980, 14, 1260-1287. [CrossRef]

9. Mikeš, J.Differential Geometry of Special Mappings; Palacký University Olomouc Print: Olomouc, Czech Republic, 2019.

10. Deshmukh, S.; Alsodais, H.; Bin Turki, N. Some results on Ricci almost solitons. Symmetry 2021, 13, 430. [CrossRef]

11. Daskalopoulos, P.; Sesum, N. The classification of locally conformally flat Yamabe solitons. Adv. Math. 2013, 240, 346-369. [CrossRef]

12. Deshmukh, S.; Alsodais, H. A note on Ricci solitons. Symmetry 2020, 12, 289. [CrossRef]

13. Turki, N.B.; Chen, B.Y.; Deshmukh, S. Conformal vector fields and Yamabe solitons. Int. J. Geom. Methods Mod. Phys. 2019, 16, 1950053. [CrossRef]

14. Suh, J.Y.; De, U.C. Yamabe solitons and Ricci solitons on almost co-Kähler manifolds. Can. Math. Bull. 2019, 62, 653-661. [CrossRef]

15. Blaga, A.M.; Ishan, A.A.; Deshmukh, S. A note on solitons with generalized geodesic vector field. Symmetry 2021, $13,1104$. [CrossRef]

16. Chen, B.-Y.; Deshmukh, S. Yamabe and quasi-Yamabe solitons on Euclidean submanifolds. Mediterr. J. Math. 2018, 15, 194. [CrossRef]

17. Burchard, A.; Mccan, R.J.; Smith, A. Explicit Yamabe flow of an asymmetric cigar. Methods Appl. Anal. 2008, 15, 65-80. [CrossRef]

18. Seko, T.; Maeta, S. Classification of almost Yamabe solitons in Euclidean spaces. J. Geom. Phys. 2019, 136, 97-103. [CrossRef]

19. Tokura, W.; Adriano, L.; Batista, E.; Bezerra, A.C. Immersion of gradient almost Yamabe solitons into warped product manifolds. arXiv 2020, arXiv:2010.03995v1.

20. Deshmukh, S.; Alsolamy, F. A note on conformal vector fields on a Riemannian manifold. Colloq. Math. 2014, 136, 65-73. [CrossRef]

21. Chow, B. The Yamabe flow on locally conformally flat manifolds with positive Ricci curvature. Commun. Pure Appl. Math. 1992, 45. 1003-1014. [CrossRef]

22. Chen, B.-Y. Total Mean Curvature and Submanifolds of Finite Type, 2nd ed.; World Scientific: Hackensack, NJ, USA, 2015.

23. Liberman, E.; Teicher, M. Homeomorphic arrangements of smooth manifolds. Symmetry 2021, 13, 981. [CrossRef]

24. Ma, L.; Cheng, L. Properties of non-compact Yamabe solitons. Ann. Glob. Anal. Geom. 2011, 40, 379-387. [CrossRef]

25. Deshmukh, S.; Turki, N. A note on $\varphi$-analytic conformal vector fields. Anal. Math. Phys. 2019, 9, 181-195. [CrossRef]

26. Hsu, S.Y. A note on compact gradient Yamabe solitons. J. Math. Anal. Appl. 2012, 388, 725-726. [CrossRef] 